\title{
FLEXIONSMARKER DER ZWEITEN PERSON SINGULAR AUS DIACHRONER UND SYNCHRONER SICHT. VOM GERMANISCHEN ZUM ALTHOCHDEUTSCHEN ZUSTAND
}

ABSTRACT: Flexion markers of 2-nd person singular from a synchronic and diachronic point of view. From the Germanic state to old-high-German

Flexion elements are a typical feature of the so-called flexion languages and are characterized by the fact that they change their form depending on (in-)flexion and they decode at least two functions. In the case of a Germanic verb inflexion elements stand for two categories: person and a singular/ plural form. In Proto-Indo-European and Proto-Germanic the inflection elements were relatively independent and it was easy to separate them from a verb stem which consisted of the so-called root and other components such as tense or mode markers. In old Germanic languages the processes of fusion and creating portmanteau word(s) (language contamination so to speak) were so advanced that it is difficult, yet not impossible, to separate primary flexion elements. The article presents the development of the 2-nd person singular marker from the Germanic state with regard to the Gothic language up to the old-high-German phase. The markers which were analysed belonged to the Present and Past Tense paradigm of all modes of the selected verb groups, that is both weak and strong ones and the rest not belonging to any of the groups mentioned above. The flexion elements were identified using a synchronic model of a morphological description worked out by Józef Darski (1987, 22004). Modification of the model concerning the character of diachronic studies ensures also a proper historical description.

KeYworDs: Gothic; Old-High-German; Inflection of the verb; Inflection endings; Alternations of core/root of the verb; Numerical category indicators; Indicators of category of person

\section{Einführung}

Die Flexionsmarker (auch: variable Exponenten oder Flexive/Flexionsendungen) sind Attribut flektierender Sprachen. Sie zeichnen sich dadurch aus, dass sie ihre Form je nach der entsprechenden Flexion ändern und mindestens zwei grammatische Funktionen kodieren. Beim germanischen Verb übernehmen die Flexionsmarker zwei

Marta Woźnicka - Uniwersytet im. Adama Mickiewicza, Poznań, marado@amu.edu.pl 
Kategorien: Person und Numerus (vgl. dazu Kotin 2012: 258). Im Urindogermanischen und Urgermanischen waren die Flexionsmarker noch relativ selbständig und ließen sich problemlos vom Verbstamm, der sich aus der Wurzel und weiteren stammbildenden Komponenten wie Tempus- und Modusmarker zusammensetzte, abgrenzen. In altgermanischen Einzelsprachen sind jedoch die Prozesse der Fusion und Kontamination im Bereich der verbalen Flexion derart fortgeschritten, dass die Abgrenzung der ursprünglichen Flexionsmarker nur schwer möglich, allerdings nicht unmöglich ist (Kotin 2012: 258). Aus genetischer und funktionaler Sicht werden die Flexionsmarker in Primär- und Sekundärendungen klassifiziert (vgl. u. a. Kluge 1906: 447-448; Prokosch 1939: §71; Krause 1968: 258-259), wobei die Primärendungen das Präsens Indikativ markieren, die Sekundärendungen dem Optativ und Aorist angehören und die Sekundärendungen eine reduzierte Form der Primärendungen darstellen (vgl. Prokosch 1939: §71; anders dazu: Kotin 2012: 260-261).

Im folgenden Beitrag wird versucht, die Entwicklung der Flexionsmarker der zweiten Person Singular vom Gotischen, das den germanischen Sprachzustand am besten dokumentiert, bis zum Althochdeutschen darzustellen. Untersucht werden die Flexionsmarker aller Verbklassen (starke, schwache Verben und besondere Verbalbildungen wie Präteritopräsentia und Reste der Verben auf -mi) im Präsens- sowie Präteritumparadigma aller Modi. Außer Acht werden die gotischen Mediopassivformen gelassen, da ihnen formal keine althochdeutschen synthetischen verbalen Formen entsprechen.

\section{Das Modell von Józef Darski und seine Anwendung in der diachronen Sprachanalyse}

Der neuhochdeutsch vorherrschende Flexionsmarker der zweiten Person Singular -st, der, außer im Imperativparadigma, die angesprochene Person markiert, ist ein Ergebnis vieler Vereinheitlichungs- und Kontaminationsprozesse, die schon im Althochdeutschen begonnen haben. Allerdings werden in verbalen Formen die Person und der Numerus nicht allein durch /st/markiert. In der Fachliteratur wird jedoch vordergründig die Endungsflexion betrachtet; stamminterne Vokal- und Konsonantenänderungen innerhalb der verbalen Formen werden, wohl aus genetischen Gründen, zwar ausführlich beschrieben, jedoch nicht als Flexionsmarker betrachtet (zu der althochdeutschen Flexion der schwachen Verben vgl. u. a. Braune/Reiffenstein 2004: 260-273, 292-303). Nicht unbegründet scheint demzufolge, zum Ziel der Festlegung der Exponenten der zweiten Person Singular, ein angemesseneres Modell der morphologischen Deskription anzuwenden, das die flektierten Verbalformen ganzheitlicher zu betrachten erlaubt. Ein tragfähiges Analyseinstrumentarium ist m. E. das synchron konzipierte Modell von Józef Darski (1987; $\left.{ }^{2} 2004\right)$, das, freilich nach seiner Modifikation bezüglich des 
diachronen Herangehens, die für das Gotische Kotin (2012) wagte, auch die historisch angemessene Beschreibung garantiert. Seine Anwendung ermöglicht es, viel präziser die verbalen Formen und ihre Komponenten in jeder Entwicklungsetappe zu beschreiben (synchrone Dimension) sowie Entwicklungstendenzen bestimmter Komponenten und des ganzen verbalen Systems aufzuzeigen (diachrone Dimension).

Im Modell von Darski beginnt die sprachliche Analyse mit der Aufspaltung der Wortformen in referenzielle Stämme und syntaktische Exponenten. Der erste Bestandteil, der referenzielle Stamm, ist relativ konstant und setzt sich im Falle von Verben aus den sich bei Konjugation (d. h. im Rahmen eines Tempus, Modus und Numerus) wiederholenden sprachlichen Mitteln zusammen. Typisch für den zweiten Bestandteil, den syntaktischen Exponenten, ist, dass er variabel ist, $d . h$. er verändert seine Form je nach der entsprechenden Person und wird daher auch als Personmarker bezeichnet. Beispielsweise gilt als Stamm im Singular des Verbs machen /max/; die Personmarker sind /ə/ für die 1., /st/ für die 2. und /t/ für die 3. Person. ${ }^{1}$ Der Stamm kann allerdings durch spezielle Zeichen markiert werden, z. B. /B/ in /li:B/ oder /A/ in /bAk/ für die Formenalternationen wie (ich) /libba/ vs. (du) /li:pst/ und (ich) /bakə/vs. (du)/bekst/, die als Indikatoren der artikulatorisch oder morphonologisch bedingten Ersatzdistribution gelten. Das Modell ist hierarchisch organisiert. Die höchste Abstraktionsstufe bildet der im Tempus, Modus und Numerus neutrale Referenzstamm, die niedrigste - der Referenzstamm im Singular (oder im Plural) desselben Tempus und Modus. Auf der niedrigsten Stufe wird von der Kategorie Person abstrahiert, wobei der Vergleich der Stämme die sog. Numerusmarker gewinnen lässt, d. h. sprachliche Elemente, die den Singular- vom Pluralstamm unterscheiden. Sind jedoch Singular- und Pluralstämme identisch, dann sind die Personmarker bifunktional, d. h. sie verweisen auf beide Kategorien: die Person und den Numerus. Aus diesem Grund werden die Person- und Numerusmarker im Folgenden nicht getrennt betrachtet und daher zusammen als Flexionsmarker (bei Kotin 2012: 225, 258-284 variable Exponenten) bezeichnet. Auf der nächsten Ebene wird von der Kategorie Numerus abstrahiert, dessen Ergebnis die sog. Modusmarker sind. Schließlich abstrahiert man von der Kategorie Modus, was die Ableitung sog. Tempusmarker ermöglicht. Als genannte Marker gelten die sprachlichen Elemente, durch die sich die jeweiligen Stämme deutlich voneinander unterscheiden. Gemeint werden jedoch im Falle der Modus- und Tempusmarker die markierten Modi (Konjunktiv und Imperativ) und Tempora (Präteritum).

${ }^{1}$ Das Modell von Darski erlaubt die Analyse auf der (ortho)graphischen wie auch phonologischen Ebene. In diesem Beitrag werden die Flexionsmarker vom Verbstamm auf der phonologischen Ebene abgetrennt, da dies den Grad an linguistischer Perfektion erreichen lässt, welcher auf der orthographischen kaum denkbar ist. Zugleich kann man dadurch neue Erkenntnisse gewinnen, die die traditionellen Ansichten stark revidieren bzw. neu bewerten lassen. 
Die für das Indogermanische bzw. Gemeingermanische charakteristische Dreimorphem-Struktur erfordert eine entsprechende Modifikation des Modells von Darski (vgl. Kotin 2012: 451-452, Anm. 82). Kotin (2012: 451-452) trennt vom Stamm den sog. stammbildenden Exponenten ab, der trotz seines möglichen ablautbedingten Formenreichtums als konstant bezeichnet wird. Als variabel wird dagegen der syntaktische Exponent bezeichnet, den Kotin, gemäß seiner Eigenschaft, als variablen Exponenten bezeichnet. Die finiten Formen des indogermanischen und gemeingermanischen Verbs weisen somit folgende Struktur auf: RVW (die referenzielle Verbalwurzel) + KE (der konstante Exponent) + VE (der variable Exponent), wobei RVW und KE zusammen den RVS (der referenzielle Verbalstamm) bilden (vgl. Kotin 2012: 225). Die konstanten Exponenten werden in zwei Gruppen eingeteilt: KE1 und KE2, wobei $\mathrm{KE} 1^{2}$, in den gängigen historischen grammatischen Darstellungen als Binde- bzw. Themavokal bekannt, Auskunft über die Verbalsemantik geben (vgl. Kotin 2012: 226) und KE2 Marker der grammatischen Kategorien (markierter Tempus: Präteritum und markierte Modi: Konjunktiv und Imperativ) sind (vgl. Kotin 2012: 226-227). Die verbale Form in der 2. P. Sg. Prät. Konj. Akt. des gotischen schwachen Verbs (Klasse II) salbôn 'salben' (salbôdedis /salboð:eð:is/ 'du salbtest') hat folgende Struktur: /salb/ $(\mathrm{RVW})+$ /o:/ (KE1) + /ðeð:/ (KE2prät.swv.) + /ii/ (KE2konj.) + /s/ (VE).

\section{Flexionsmarker der 2. Person Singular}

\subsection{Flexionsmarker der 2. Person Singular im Gotischen}

Als genuine, aus dem Indogermanischen und Gemeingermanischen vererbte Flexionsmarker der zweiten Person Singular gelten im Gotischen /s/, /t/ und der sog. Nullexponent, wobei /s/ bis zu drei unterschiedliche Quellen hat (vgl. Kotin 2012: 270-272): (1) /s/ (idg. *-si, germ. *-si/*-zi) tritt im Präsens Indikativ Aktiv der starken Verben (stV.) $)^{3}$, schwachen Verben (swV.) ${ }^{4}$, bei sein und wollen auf, z. B. starke Verben: /gr'i:p1s/ 'du greifst', /g'iß1s/ 'du gibst', /sl'e:pıs/ 'du schläfst', /h'aitis/ 'du heißt', /gr'e:tıs/ 'du weinst, du klagst'; schwache Verben: /n'asjis/ 'du heilst', 'du rettest', /l'ßibais/ 'du lebst'; /is/ 'du bist', /w'ilirs/ 'du willst'.

(2) /s/ eines anderen Ursprungs (idg. ${ }_{-s}$, germ. $\left.{ }_{-}^{*} /{ }^{*}-z\right)$ kommt dagegen im Präsens Konjunktiv Aktiv der starken, schwachen Verben und beim Verb sein sowie im Präteritum Konjunktiv Aktiv derselben Gruppen von Verben und bei Präteritopräsentia vor, z. B.:

\footnotetext{
${ }^{2}$ Zur Spezifikation der KE1 vgl. Kotin 2012: 227-250.

${ }^{3}$ Gemeint werden hier alle Gruppen der starken Verben, d. h. I. die ablautenden, II. die reduplizierenden und III. die ablautend-reduplizierenden Verben.

${ }^{4}$ Gemeint werden alle Klassen der schwachen Verben, d. h. jan-, ôn-, ân- und nan-Verben.
} 
Präsens Konjunktiv Aktiv

starke Verben:

/n'iutais/ 'du treffest, du erreichest'

/s'exvais/' 'du sehest'

/h'aldais/ 'du hütest'

/gr'e:tais] 'du weinest'

schwache Verben:

/br'añjais/ 'du brennest'

/s'albo:s/ 'du salbest'

/fulnais/ 'du werdest voll'

/s'ijais/ 'du seiest'

\author{
Präteritum Konjunktiv Aktiv \\ starke Verben: \\ /fr'o:yis/ / 'du fragtest' \\ /bebl'o:tis/ 'du verehrtest' \\ /gegr'o:ti:s/ 'du weintest' \\ schwache Verben: \\ /d'ailðıеð:is/ 'du teiltest' \\ /s'alboð:eð:is/ / 'du salbtest' \\ /Br'laiðeð:is/ 'du lebtest' \\ /w'e:siss/ 'du wärest' \\ Präteritopräsentia: \\ /w'ise:dis/ ' 'du wüsstest' \\ /'Exte:diss/ 'du hättest'
}

Schließlich stammt der Flexionsmarker der gotischen schwachen präteritalen Verbalformen im Indikativ (/ðe:s/ wie in /n'asðre:s/ 'du rettetest' oder /l'ıßaiðe:s/ 'du lebtest') von der Personalendung des indogermanischen medialen Aorist (idg. *-thês, germ. *-đêz) (vgl. Kotin 2012: 272). Das /ð/ in gotischen verbalen Formen wird jedoch auch als eindeutiger Marker des Präteritums interpretiert und es wird auch vermutet, dass der indogermanische Formant des Aorist mögliche Quelle des germanischen schwachen Präteritums ist. Kotin (2012: 272) gesteht dabei zu, dass die einzig mögliche, wohl aber nicht zufriedenstellende Interpretation ist, dass es im Falle von diesen Formen zur Kontamination des dentalen Bestandteils des aoristischen Formanten und des Präteritummarkers gekommen ist. Unklar sei dagegen der Status von /e:/ in /ðe:s/, den Kotin (2012: 272) sowohl für den Bestandteil des Präteritum- als auch des Flexionsmarkers hält. Als eindeutiger Marker der 2. Person Singular gilt gewiss /s/ in diesen Formen.

Im Präteritum Indikativ Aktiv der starken Verben sowie im Präsens Indikativ Aktiv der Präteritopräsentien gilt als Flexionsmarker der 2. Person Singular $/ t /$, das genuine Perfektendung ist (idg. *-tha, germ. *- $\left.p /{ }^{*}-t\right)$, z. B.: starke Verben: /fro: $\mathbf{t}$ / 'du fragtest', /heh'alst/ 'du hütetest', /gegr'o:st/ 'du weintest', /s\&s'o:t/ 'du sätest's; Präteritopräsentien: /mart/ 'du kannst'.

Synchron für das Gotische gesehen müssen auch neben /t/ als Flexionsmarker der 2. Person Singular im Präteritum Indikativ Aktiv der starken Verben und im Präsens Indikativ Aktiv der Präteritopräsentia folgende formale Änderungen betrachtet werden:

${ }^{5}$ Bei Verben haldan (/heh'alst/) und gretan (/gegr'o:st/) kann synchron gesehen /st/ als Flexionsmarker betrachtet werden. /st/ wird in der 2. Person Singular der starken Verben festgestellt, die nach dem Weglassen des /an/ evtl. /jan/ beim Infinitiv als letzten Laut einen dentalen Verschluss- (/t/ evtl. /d/) oder

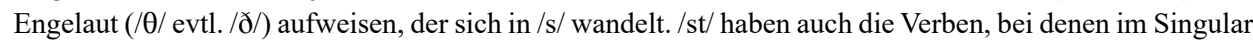
der 1 . und 3. Person der automatische Wechsel / $/ / \mathrm{zu} / \theta /$ festzustellen ist. 
1. der Vokalwechsel', z. B.: starke Verben: /waixxt/ 'du kämpftest', /afl'aisst/ 'du gingst weg', / 1 launxt/ 'du flohst', /gaust/ 'du gossest/gosst', /warpt/ 'du warfst', /fanst/ 'du erkanntest',/namt/ 'du nahmst', /bast/ 'du batest'; Präteritopräsentien: /kant/ 'du weißt/ kennst', /waist/ 'du weißt'?

2. der Vokalwechsel zusammen mit dem auslautbedingten Wandel $/ \beta /$ zu $/ \Phi /$, der im Singular aller drei Person zum Ausdruck kommt, z. B.: /drai $\phi \mathbf{t}$ / 'du triebst', /afsk' au $\Phi \mathbf{t}$ / 'du verstießest/verstießt', /gaфt/ 'du gabst'.

Die genuine Imperativform, d. h. die der 2. Person Singular ${ }^{8}$ weist im Gotischen den sog. Nullexponenten als Flexionsmarker auf, z. B.: starke Verben: /nım-ø/ 'nimm!', /sle:p-ø/ 'schlaf(e)!',/sai-ø/ ‘säe!'; schwache Verben:/dailii-ø/ 'teil(e)!', /l‘rßai-ø/ ‘leb(e)!' . Phonologisch gesehen gilt jedoch auch als Marker der 2. Person Singular im Imperativparadigma bestimmter gotischer starker Verben die Verhärtung der stimmhaften Laute, nämlich / $/ \mathrm{d} / \mathrm{zu} / \theta /$ und $/ \beta / \mathrm{zu} / \phi /$ im Auslaut, z. B.: /g'awi $\theta /$ 'binde zusammen!', /skai $\theta /$ 'trenne (dich)!', /gar'e: $\theta /$ 'triff Vorsorge!', /dri: $\phi /$ 'treib(e)!', /g1 $\phi /$ ' 'gib!', /gra $\Phi$ / 'grab(e)!'.

\subsection{Flexionsmarker der 2. Person Singular im Althochdeutschen}

Das /s/ als Marker der 2. Person Singular wird auch in althochdeutschen verbalen Formen realisiert, allerdings wird der Exponent seit dem 9. Jahrhundert um /t/ ergänzt, wessen Ergebnis der in gegenwärtigen verbalen Formen vorherrschende Marker/st/ ist. Entstanden ist der Flexionsmarker aus den Formen mit enklitisch angefügtem thu, $d u$, in denen sich der dental $(t h, d)$ dem $s$ anschloss. Vermutlich haben bei der Entstehung des besprochenen Flexivs auch die Formen der Präteritopräsentia wie kanst oder muost u. a. mitgewirkt (vgl. Braune/Reiffenstein 2004: 261). /s/, später/st/ ist im Präsens Indikativ sowie Konjunktiv Aktiv der starken und schwachen Verben sowie der Verben tuon, gān, stān und wollen (nur im Präsens Konjunktiv Aktiv) vorhanden.

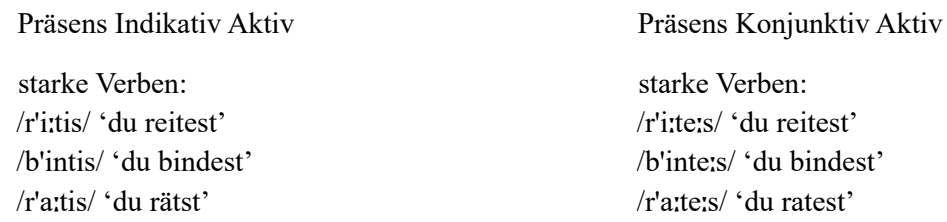

${ }^{6}$ Die Vokalwechsel sind: 1. /ai/ zu /1/ evtl. /\&/ vor /r/, /x/ oder /xv/, 2. /au/ zu /u/ evtl. / / / vor /r/, /x/ $\operatorname{oder} / \mathrm{xv} /$, 3. /a/ zu / / / evtl. /o/ vor/r/, /x/ oder/xv/, 4. /a/ zu /e:/. Die Vokalwechsel sind dem gewählten Modell der morphologischen Deskription nach auch Marker des markierten Tempus, d. h. des Präteritums.

${ }^{7} \mathrm{Zu} / \mathrm{st} /$ in den Formen /afl'aist/ (weggehen), /gaust/ (gießen), /fanst/ (erkennen), /bast/ (bitten) und /waist/ 'du weißt' vgl. die Anmerkung Nr. 5 in diesem Beitrag.

${ }^{8} \mathrm{Zu}$ den indogermanischen Spendeformen für den germanischen Imperativ vgl. Kotin 2012: 256-258. Zur Entwicklung des Imperativmarkers im Deutschen vgl. Woźnicka 2014: 157-165. 
schwache Verben:

/t'eilis/ 'du teilst'

/s'albo:s/ 'du salbst'

/l'ebe:s/ 'du lebst'

/tuos/ 'du tust'

/ga:s/ evtl. /ge:s/ 'du gehst'

/sta:s/ evtl. /ste:s/ 'du stehst'

\author{
schwache Verben: \\ /t'eilie:s/ 'du teilest' \\ /s'albo:s/ evtl. /s'albo:e:s/ 'du salbest' \\ /l'zbe:s/ evtl. /l'Ebe:e:s/ 'du lebst' \\ /tues/ 'du tuest' \\ /ge:s/ 'du gehest' \\ /ste:s/'du stehest' \\ /w'Ele:s/ 'du wollest'
}

Ferner tritt/s/ oder /st/ im Präteritum Indikativ und Konjunktiv Aktiv der schwachen Verben, Präteritopräsentia und des Verbs wollen sowie im Präteritum Konjunktiv Aktiv der starken Verben und des Verbs wollen.

$\begin{array}{ll}\text { Präteritum Indikativ Aktiv } & \text { Präteritum Konjunktiv Aktiv } \\ & \text { starke Verben: } \\ & \text { /r'itiss/ 'du rittest' } \\ & \text { /b'untiss/ 'du bändest' } \\ & \text { /h'iasi:s/ 'du hießest' } \\ & \text { schwache Verben: } \\ \text { schwache Verben: } & \text { /t'eiltiss/ 'du teiltest' } \\ \text { /t'eilto:s/ 'du teiltest' } & \text { /s'albo:ti:s/ 'du salbtest' } \\ \text { /s'albo:to:s/ 'du salbtest' } & \text { /l'Ebe:tiss/ 'du lebtest' } \\ \text { /l'Ebe:to:s/ 'du lebtest' } & \text { Präteritopräsentia: } \\ \text { Präteritopräsentia: } & \text { /w'isti:s/ 'du wüsstest' } \\ \text { /w'īso:s/ evtl. /w'isto:s/ 'du wusstest' } & \text { /k'ondi:s/ 'du wüsstest/könntest' } \\ \text { /k'ondo:s/ 'du wusstest/konntest' } & \text { /sk'olti:s/ 'du solltest' } \\ \text { /sk'olto:s/ 'du solltest' } & \\ & \text { /t'artiss/ 'du tätest' } \\ & \\ & \text { /w'oltiss/ 'du möchtest' }\end{array}$

Nebst /s/ bzw. /st/ kann als Marker der 2. Person Singular im Präsens Indikativ Aktiv in der Gruppe starker Verben auch der Stammvokalwechsel ${ }^{9}$ funktionieren.

$\begin{array}{llllll}\text { st.V. II } & \text { st.V. IIIb } & \text { st.V. IV } & \text { st.V.V } & \text { st.V. VI } & \text { st.V. VII1a } \\ \text { /l'iugis/ } & \text { /v'irfis/ } & \text { /st'ilis/ } & \text { /g'ibis/ } & \text { /f'eris/ } & \text { /h'eltis/ } \\ \text { 'du lügst' } & \text { 'du wirfst' } & \text { 'du stiehlst' } & \text { 'du gibst' } & \text { 'du fährst' } & \text { 'du hältst' }\end{array}$

\footnotetext{
${ }^{9}$ Gemeint werden die starken Verben: 1. der Klasse II, die vor /a e o/ der Folgesilbe, d. h. im Plural /io/ als Stammvokal, ansonsten /iu/ aufweisen; 2. der Klasse IIIb sowie IV und V, die im Singular/i/ und im Plural

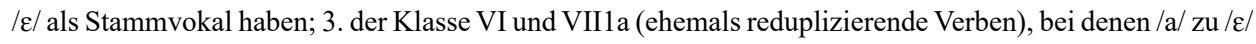
in der 2. und 3. Person Singular umgelautet wird, wobei im letzteren Fall auch verbale Formen vorhanden sind, in denen der Stammvokal nicht umgelautet wird, z. B.: /sl'axis/ 'du schlägst' oder /h'altis/ 'du hältst'.
} 
Die alte Perfektendung /t/ eventuell /t/ samt dem Vokalwechsel, die im Gotischen den Formen der 2. Person Singular der starken Verben im Präteritum Indikativ Aktiv sowie der Präteritopräsentia im Präsens Indikativ Aktiv als dessen Flexionsmarker zugehörte, weisen im Althochdeutschen lediglich Präteritopräsentia im Präsens Indikativ Aktiv auf, z. B.: /weist/ 'du weißt', /kanst/ 'du weißt/verstehst/kannst', /darft/ 'du hast es nötig', /skalt/ 'du sollst', /maxt/ 'du kannst/vermagst', /muost/ 'du hast Gelegenheit/magst’.

Im Präteritum Indikativ Aktiv weisen die starken Verben /i/ oder /i/ zusammen mit dem Vokalwechsel und resp. dem grammatischen Wechsel als Marker der 2. Person Singular auf, da die Westgermania im Unterschied zum Ost- und Nordgermanischen den Exponenten hat, der aus dem starken (thematischen) Aorist stammt (vgl. Kotin 2012: 271), z. B.: /r'iti/ 'du rittest', /ts 'igi/ 'du ziehst/zeihtest', /l'ugi/ 'du logst', /b 'uti/ 'du botest', /s'uti/ 'du sottest/siedetest', /b'unti/ 'du bandest', /v'urfi/ 'du warfst', /v'urti/ 'du wurdest', /st'arli/ 'du stahlst',/g'arbi/ 'du gabst', /l'arri/ 'du last/lasest',/f'uori/ ‘du fuhrst', /sl' 'uogi/ 'du schlugst', /h' ialti/ 'du hieltst'. Das /i/samt dem Vokalwechsel gilt auch als Flexionsmarker der angesprochenen Person im Singular beim Verb wollen im Präsens Indikativ Aktiv (/w'ili/) und beim Verb tuon im Präteritum Indikativ Aktiv $\left(/ t^{c} a t^{2} \mathrm{i} /\right)$.

Im Imperativparadigma übernimmt die Person- und Numeruskennzeichnung entweder der sog. Nullexponent oder der Stammvokalwechsel. Den Nullexponenten weisen alle schwachen Verben, die starken Verben der Klassen I, IIIa, VI und VII sowie das Verb tuon auf, z. B.: schwache Verben: /br' $\varepsilon n i-\varnothing /$ 'brenne!', /t'eili-ø/ ‘teil(e)!', /s'albo-ø/ 'salb(e)!', /l'عbe-ø/ 'leb(e)!'; starke Verben: /ritt-ø/ 'reite!', /bint-ø/ 'binde!' , /far-ø/ 'fahr(e)!', /halt-ø/ 'halt!', /tuo-ø/ 'tue!'; den Vokalwechsel dagegen die starken Verben der Klassen II, IIIb, IV und V sowie das Verbum substantivum, z. B.: /liug/ 'lüg(e)!', /hiuf/ 'klag(e)!', /biut/ 'biete!', /virf/ 'wirf!', /brix/ 'brich!', /gib/ 'gib!', /wis/ 'sei!'.

\section{Fazit}

Resümierend kann festgestellt werden, dass der Flexionsmarker/s/ (im Althochdeutschen seit dem 9. Jahrhundert/st/) sowohl im Gotischen als auch im Althochdeutschen in denselben Paradigmen realisiert wird, d. h. im Präsens Indikativ und Konjunktiv Aktiv sowie im Präteritum Indikativ (nur in der Gruppe der schwachen Verben, wobei im Althochdeutschen noch bei Präteritopräsentia und wollen) und Konjunktiv Aktiv. Im Althochdeutschen wird auch zusammen mit/s/ evtl. /st/ im Falle von bestimmten starken Verben im Präsens Indikativ Aktiv der stamminterne Vokalwechsel als Marker der angesprochenen Person im Singular betrachtet, z. B. /v'irfis/ 'du wirfst'. Das gotische /t/ (z. B. /froy:t/ 'du fragtest'), das gegebenenfalls mit dem Vokalwechsel (z. B. /warpt/ 'du warfst'), /kant/ 'du weißt/kannst') und dem auslautbedingten Wechsel / $\beta /$ $\mathrm{zu} / \phi /$ (z. B. /ga $\Phi \mathrm{t} /$ 'du gabst') die 2. Person Singular im Präteritum (starke Verben) und 
Präsens (Präteritopräsentia) Indikativ Aktiv markiert, ist in althochdeutschen verbalen Formen nur im Präsens Indikativ Aktiv der Präteritopräsentia vorhanden, z. B. /muost/ 'du hast Gelegenheit, magst', /darft/ 'du hast nötig, bedarfst'. In althochdeutschen Verbformen (Präteritum Indikativ Aktiv der starken Verben und des Verbs tuon sowie Präsens Indikativ Aktiv des Verbs wollen) ist dagegen der aus dem Flexionssystem des indogermanischen starken (thematischen) Aorist stammende und beim Übergang zum Mittelhochdeutschen bis zum Schwa-Laut hin reduzierte Exponent/i/ anzutreffen, z. B. /f'uori/ 'du fuhrst', der samt dem Vokalwechsel innerhalb des Stammes (z. B. /v'urfi/ 'du warfst', /t' atti/ 'du tatest', /w'ili// 'du willst') eventuell auch dem Wechsel des stammschließenden Konsonanten (Stichwort: grammatischer Wechsel, z. B. /s'uti/ 'du sottest') als Flexionsmarker funktioniert. Im Imperativparadigma dominiert sowohl im Gotischen als auch im Althochdeutschen der sog. Nullexponent als Flexionsmarker und zwar in der Gruppe der starken wie auch der schwachen Verben, z. B. got. /sle:p-ø/ 'schlaf(e)!', /d'ailii-ø/ 'teil(e)!' und ahd. /ritt-ø/ 'reite!', /t'eili-ø/ 'teil(e)!'. Allerdings markieren die 2. Person Singular im Imperativ auch Stammesveränderungen: im Gotischen die auslautbedingte Verhärtung des stammschließenden Konsonanten $(/ \beta / \mathrm{zu} / \phi /$ und $/ ð /$ zu $/ \theta /$, z. B. /gar' $e: \theta /$ 'triff Vorsorge!', /dri: $\Phi /$ 'treib(e)!'); im Althochdeutschen der stamminterne Vokalwechsel, z. B. /virf/ 'wirf!'.

Die präsentierten Ergebnisse zeugen davon, dass eine widerspruchsfreie und exakte Ermittlung der Flexionsmarker der 2. Person Singular wie auch weiterer Personen im Singular- und Pluralparadigma, sowie ihre diachrone Analyse, auch unter Berücksichtigung der verbalen Formen in weiteren Entwicklungsetappen des Deutschen, mit dem von Darski zur Verfügung gestellten Analyseinstrumentarium durchaus durchführbar sind. Der universale Charakter des Darski-Modells ermöglicht sowohl eine synchrone als auch diachrone Analyse des Sprachmaterials, ohne dass ihre Ergebnisse widersprüchlich sind. Im Endeffekt erlaubt es auch festzustellen, in welchen Sprachstufen der Wandel im verbalen Bereich im hohen Maße archaische Züge und in welchen vorwiegend neuartige Merkmale aufweist, was zweifelsohne Einblick in die zukünftige Entwicklungsrichtung der verbalen Flexion geben kann.

\section{Literatur}

Braune, Wilhelm (2004): Althochdeutsche Grammatik I. Laut- und Formenlehre. 15. Auflage, bearbeitet von Ingo Reiffenstein. Tübingen: Max Niemeyer Verlag.

Braune, Wilhelm (2004): Gotische Grammatik. 20. Auflage, bearbeitet von Frank Heidermanns. Tübingen: Max Niemeyer Verlag.

Darski, Józef (1987): Linguistisches Analysemodell. Definitionen grundlegender grammatischer Begriffe. Poznań: Wydawnictwo Naukowe UAM.

Darski, Józef (2004): Linguistisches Analysemodell. Definitionen grundlegender grammatischer Begriffe. 2., völlig neu bearbeitete und ergänzte Auflage. Poznań: Wydawnictwo Naukowe UAM.

Darski, Józef (2010): Deutsche Grammatik. Ein völlig neuer Ansatz. Frankfurt a. M. u. a.: Peter Lang Verlag. 
Kluge, Friederich (1906): Vorgeschichte der altgermanischen Dialekte. 2. Auflage. Straßburg: Karl J. Trübner.

Kotin, Michail L. (2012): Gotisch: Im (diachronischen und typologischen) Vergleich. Heidelberg: Winter. Krause, Wolfgang (1968): Handbuch des Gotischen. 3. Auflage. München: Beck.

Prokosch, Ernst (1939): A Comparative Germanic Grammar. Philadelphia: Linguistic Society of America. Woźnicka, Marta (2014): „Zum Wandel in den Exponenten im Imperativparadigma vom Gotischen bis zum Neuhochdeutschen." In: Jokiel, Małgorzata/Pelka, Daniela (Hgg.): Germanistische Werkstatt 6. Opole: Wydawnictwo Uniwersytetu Opolskiego. 157-165. 